\title{
Non-Target Enhancing Lesion Response
}

National Cancer Institute

\section{Source}

National Cancer Institute. Non-Target Enhancing Lesion Response. NCI Thesaurus. Code C135480.

An assessment of the response to the therapy of the non-target enhancing lesion. 